Classification

Physics Abstracts

$64.70 \mathrm{~K}$

\title{
Neutron diffraction study of sodium nitrite in an applied electric field
}

\author{
D. Durand, F. Denoyer, D. Lefur \\ Laboratoire de Physique des Solides (*) \\ Université Paris-Sud, Bâtiment 510, F-91405 Orsay, France \\ R. Currat and L. Bernard \\ Institut Laue-Langevin, 156X, 38042 Grenoble Cedex, France
}

(Reçu le 16 décembre 1982, accepté le 17 janvier 1983)

\begin{abstract}
Résumé. - Le diagramme de phase de $\mathrm{NaNO}_{2}$ sous champ électrique est exploré par diffraction neutronique. On n'observe pas de phases commensurables induites par le champ. Pour un champ de $3,4 \mathrm{kV} \mathrm{cm}^{-1}$ on observe un point triple où les trois phases paraélectrique, ferroélectrique et modulée coexistent. Les résultats sont discutés sur la base d'un modèle phénoménologique à la Landau.
\end{abstract}

\begin{abstract}
The phase diagram of $\mathrm{NaNO}_{2}$ in an applied electric field is investigated by means of neutron diffraction. No evidence is found for field-induced commensurate phases. For an applied field of $3.4 \mathrm{kV} \mathrm{cm}^{-1}$ a triple point is observed at which the paraelectric, ferroelectric and modulated phases coexist. Results are discussed in terms of a phenomenological Landau free-energy.
\end{abstract}

1. Introduction. - Sodium nitrite has been the subject of a considerable number of experimental [1-5] and theoretical [6-12] studies. Between the high-temperature $\left(T>T_{\mathrm{i}} \simeq 164^{\circ} \mathrm{C}\right)$ paraelectric phase (space group Immm) and the low temperature $\left(T<T_{\mathrm{f}} \simeq 162.5^{\circ} \mathrm{C}\right)$ ferroelectric phase (space group $\operatorname{Im} 2 \mathrm{~m}$ ), $\mathrm{NaNO}_{2}$ exhibits a sinusoidally-modulated structure, characterized by a polarization wave of wavevector

$$
\mathbf{q}_{\delta}=\delta \mathbf{a}^{*} .
$$

The temperature dependence of $\delta$, between $T_{\mathrm{i}}$ and $T_{\mathrm{f}}$, as obtained from a recent neutron diffraction study [13], shows a smooth decrease from $\delta\left(T_{\mathrm{i}}\right) \simeq 0.122$ to $\delta\left(T_{\mathrm{f}}\right) \simeq 0.101$, with no evidence for intermediate " lock-in " phases at rational values of $\delta$ (e.g. $\delta=2 / 17,1 / 9,2 / 19)$. In the same study, no evidence for higher order satellite reflections could be detected, thus confirming the sinusoidal character of the polarization wave. Also, hysteresis effects were found to be limited to the vicinity of the first-order transition at $T_{\mathrm{f}}$.

(*) Associé au CNRS. 
This behaviour is in contrast to that found in thiourea, another ferroelectric compound which exhibits several intermediate modulated structures sandwiched between the high temperature paraelectric and the low-temperature ferroelectric phases. X-ray and neutron studies of thiourea $[14,15]$ as a function of temperature and pressure have yielded extensive evidence for the occurrence of non-sinusoidal modulations and large wavevector hysteresis.

Not surprisingly, though not necessarily for the same reasons, the two compounds appear to behave differently under an applied d.c. electric field. Birefringence measurements on thiourea [16] indicate $\mathrm{a}(E, T)$ phase diagram of the type shown in figure $1 a$. The stability region for the modulated phase is limited by a first-order transition line from $T_{\mathrm{f}}$ to $T_{\mathrm{tr}}$ and a second-order transition line from $T_{\mathrm{tr}}$ to $T_{\mathrm{i}}$. Additional susceptibility anomalies within the modulated region, have been shown $[17,18]$ to correspond to a field-induced commensurate lock-in phase of wavevector $b^{*} / 8$. On the other hand dielectric susceptibility measurements by Gesi [19] on $\mathrm{NaNO}_{2}$ suggest a phase diagram as shown in figure $1 b$, i.e. with a triple point at $\left(E_{\mathrm{T}}, T_{\mathrm{T}}\right)$ and a critical end point at $\left(E_{\mathrm{c}}, T_{\mathrm{c}}\right)$. No details are given in reference [19], concerning the order of the various transition lines, although the topology of the phase diagram would seem to imply the existence of a tricritical point somewhere between $T_{\mathrm{i}}$ and $T_{\mathrm{T}}$.

The purpose of the present study is to reinvestigate the $(E, T)$ phase diagram of $\mathrm{NaNO}_{2}$ by means of neutron diffraction. The main advantage of the latter technique, as compared to macroscopic ones, lies in the fact that it yields useful informations about the $E$-and $T$-dependence of the amplitude, wavevector and character of the wave.
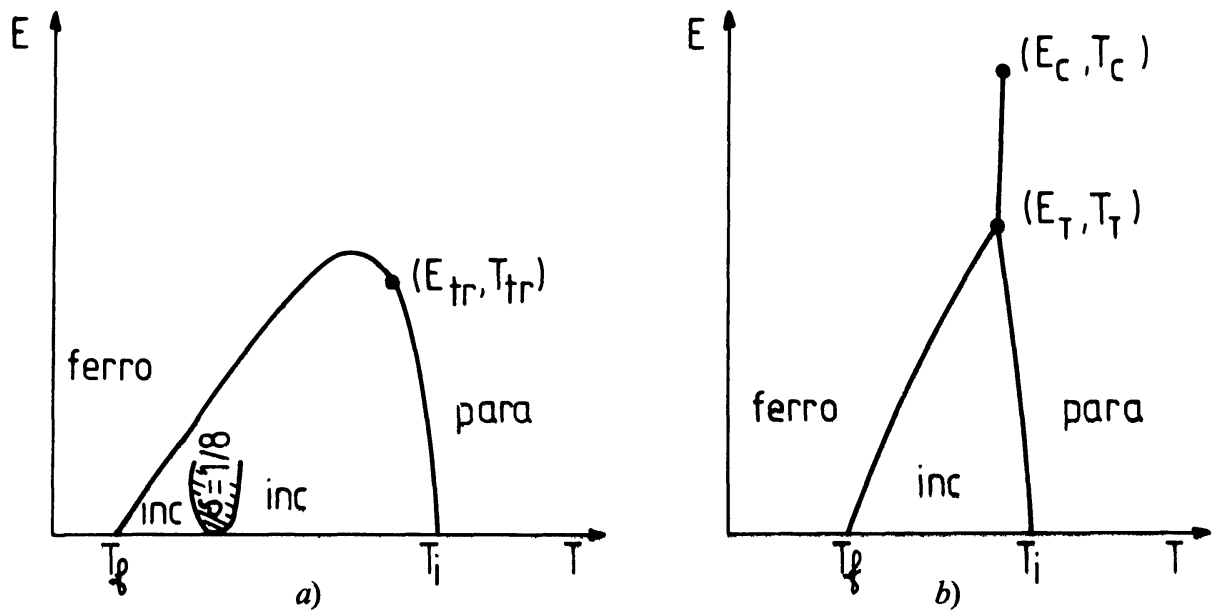

Fig. 1. - Schematic $(E, T)$ phase diagram observed in $(a)$ thiourea [16-18] and $(b)$ sodium nitrite [19]. In both cases the electric field is applied along the ferroelectric axis.

2. Experimental. - The measurements were performed on the IN8 3-axis spectrometer at the Institut Laue-Langevin, using a PG-filtered incident neutron wavelength of $2.36 \AA$.

The specimen is a $1.5 \times 8 \times 8 \mathrm{~mm}^{3}$ plate cut from a single crystal boule, grown by Professor J. P. Chapelle (SDTM, LP 3261, Université Paris-Sud, 91405 Orsay). A $1 \mu$ Cu-film has been sputtered onto the large faces $(\perp$ b) of the crystal plate. The specimen is mounted in a $30 \mathrm{~W}$ electrical furnace designed to provide a temperature homogeneity of a few $10^{-2} \mathrm{~K}$ inside the sample chamber. The temperature stability, averaged over several hours, is found to be better than $0.01 \mathrm{~K}$. Electric field inhomogeneities were minimized by purposely reducing the neutron beam crosssection in such a way as to mask off the periphery of the specimen.

The aim of the experiment is to monitor the intensity and position of the strong $( \pm \delta, 2,0)$ 
satellite reflections, as a function of temperature and electric field, independently. In practice a major difficulty arises from the finite electrical conductivity of the specimen $\left(\rho=2.4 \times 10^{6} \Omega \mathrm{m}\right.$ at $164^{\circ} \mathrm{C}$ ), giving rise to Joule heating under an applied electric field. The heat generated in the bulk of the specimen, a few $\mathrm{mW}$ at large fields, diffuse outwards and creates a temperature difference of up to $0.5 \mathrm{~K}$ between the specimen and the sensor. Note that thermal gradients within the specimen remain negligible because of the thin plate-like shape of the latter with large metallized faces. Since the thermal resistance between specimen and sensor can be taken as independent of field and temperature (over a range of a few $\mathrm{K}$ ) the temperature difference between the two may be written as :

$$
\Delta T=k V I,
$$

where $V$ and $I$ stand for the measured voltage and current across the specimen. The value of the proportionality constant $k$ was adjusted empirically $\left(k \simeq 0.13 \mathrm{~mW}^{-1} \mathrm{~K}\right)$ as discussed below. The temperature scale for the data shown in figure 2 , has been corrected according to equation (1) above (the correction is approximately proportional to $E^{2}$ ).

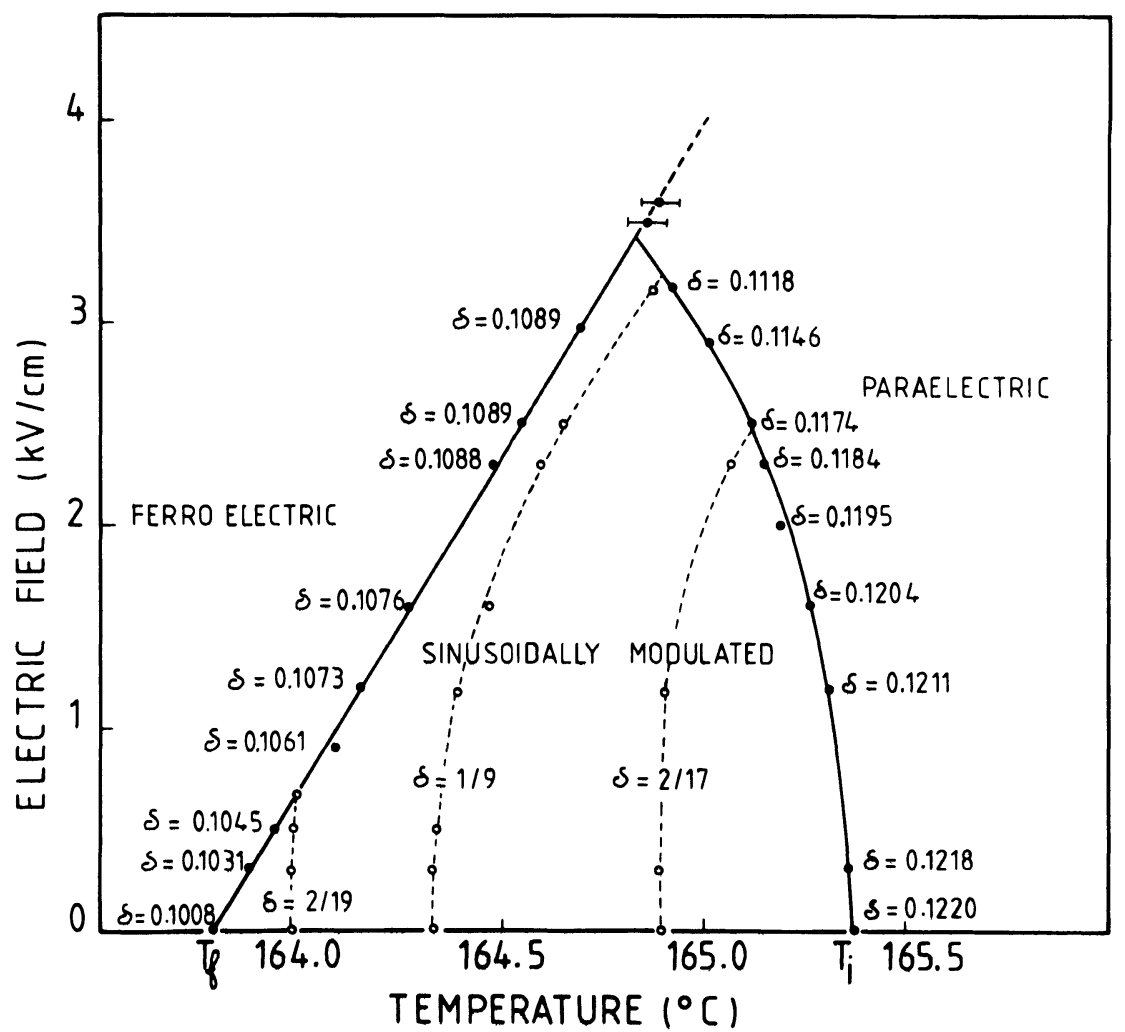

Fig. 2. - Observed $(E, T)$ phase diagram of $\mathrm{NaNO}_{2}$. The commensurate iso- $\delta$ lines are shown as dotted lines.

3. Results. - 3.1 PHASE BOUNDARIES. - Figure 2 summarizes our results concerning the position of the paraelectric-to-modulated transition line $T_{\mathrm{i}}(E)$ and of the modulated-to-ferroelectric line $T_{\mathrm{f}}(E)$. Strong critical scattering is observed near the $T_{\mathrm{i}}(E)$ line, indicating a continuous transition all the way up to the triple point $\left(T_{\mathrm{T}}=164.8^{\circ} \mathrm{C}, E_{\mathrm{T}}=3.4 \mathrm{kV} / \mathrm{cm}\right)$. The tran- 
sition at $T_{\mathrm{f}}(E)$ is clearly discontinuous as evidenced by both the abrupt vanishing of the satellite intensity and the simultaneous observation of a discontinuity in the sample's electrical resistance ( $V / I$ ratio). The initial slope of the $T_{\mathrm{f}}(E)$ line is found to be :

$$
\left[\frac{\mathrm{d} E}{\mathrm{~d} T_{\mathrm{f}}}\right]_{E=0}=3.26 \mathrm{kV} \mathrm{cm}^{-1} \mathrm{~K}^{-1} \text {. }
$$

This result is independent of the value chosen for the coefficient $k$ in equation (1). However at finite field values the position of the $T_{\mathrm{i}}(E)$ and $T_{\mathrm{f}}(E)$ lines depend upon $k$. In fact, the value of $k$ was chosen in such a way as to obtain a straight line for the $T_{\mathrm{f}}(E)$ line. This criterion will be justified on the basis of the model developed in $\S 4$.

Above $E_{\mathrm{T}}$, no satellite reflections are observed. However, a resistivity anomaly can still be detected indicating a weakly first-order direct transition between paraelectric and ferroelectric phases. The location of the critical end-point could not be determined in the present study.

Our results are in quantitative disagreement with those of Gesi [19], which indicate a triple point at a lower field value $\left(\simeq 2.6 \mathrm{kV} \mathrm{cm}^{-1}\right)$. Too few details are given in reference [19] about measuring conditions for us to make a plausible guess as to the origin of this discrepancy.

3.2 Field-DEPENDENCE OF MODUlation WAVEVECTOR. - Also shown in figure 2 are the values $\delta_{\mathrm{i}}(E)$ and $\delta_{\mathrm{f}}(E)$ of the modulation wavevector in reduced units, measured along the $T_{\mathrm{i}}(E)$ and $T_{\mathrm{f}}(E)$ lines, respectively. For fields up to $2.5 \mathrm{kV} / \mathrm{cm}$ we find that the quantity $\delta_{\mathrm{i}}^{2}(E)-\delta_{\mathrm{i}}^{2}(0)$ varies quadratically with $E$ :

with

$$
\delta_{\mathrm{i}}^{2}(E)-\delta_{\mathrm{i}}^{2}(0) \simeq \lambda E^{2}
$$

$$
\lambda=-(1.6 \pm 0.2) \times 10^{-4}(\text { r.u. })^{2} \mathrm{kV}^{-2} \mathrm{~cm}^{2} \text {. }
$$

At the triple point we have :

$$
\delta_{\mathrm{i}}\left(E_{\mathrm{T}}\right)=\delta_{\mathrm{f}}\left(E_{\mathrm{T}}\right)=0.109 .
$$

Within the modulated region the modulation wavevector appears to vary smoothly as a function of field and temperature. No evidence is found for a plateau at the commensurate values $\delta=2 / 17,1 / 9,2 / 19$ (dotted lines in Fig. 2). This is in contrast to the case of thiourea where a fieldinduced commensurate phase at $\delta=1 / 8$ is observed. This difference is readily understood on the basis of the following symmetry argument. The lowest order candidate for a lock-in energy term at, say, $\delta=1 / 9$ has the form

$$
V^{(9)} P_{\mathrm{q}_{\delta}}^{9}+\text { c.c. , }
$$

where $P_{\mathrm{q} \delta}$ is the (complex) amplitude of the modulation and $V^{(9)}$ is an appropriate anharmonic coefficient. In the present case, the term written above is forbidden for 2 reasons :

i) The quantity $P_{\mathrm{q}_{\delta}}^{9}$ is not rotationally invariant.

ii) The first Umklapp vector along $\mathbf{a}^{*}$ is $2 \mathbf{a}^{*}$ (due to the body-centred nature of the hightemperature structure). Thus for $\mathbf{q}_{\delta}=\mathbf{a}^{*} / 9$ the above term is not translationally invariant.

Applying an electric field induces a new potential lock-in term of the form :

$$
V^{(10)} P_{0} P_{\mathrm{q}_{\delta}}^{9}+\text { c.c. , }
$$

where $P_{0}$ is the amplitude of the homogeneous polarization. This type of cross-term is believed to stabilize the $\delta=1 / 8$ phase in thiourea [15]. Here, however, it is still translationally forbidden. Hence, one must resort to the next higher-order term :

$$
V^{(18)} P_{q_{\delta}}^{18}+\text { c.c. . }
$$

Such a term is allowed, with or without field, but is obviously too weak to be effective. 
3.3 AMPLITUdE AND CHARACTER OF THE MODULATION. - The study of the first-order satellite intensity shows that the order parameter varies monotonically as a function of temperature and electric field, the iso-intensity curves being roughly parallel to the second-order line $T_{\mathrm{i}}(E)$.

Finally we find that the applied electric field does not alter the sinusoidal character of the modulation. This point is illustrated in figure 3 which shows that at $2.3 \mathrm{kV} / \mathrm{cm}$ the second- and thirdorder satellite reflections are too weak to be detected. This observation is at variance with the prediction made in reference [8].

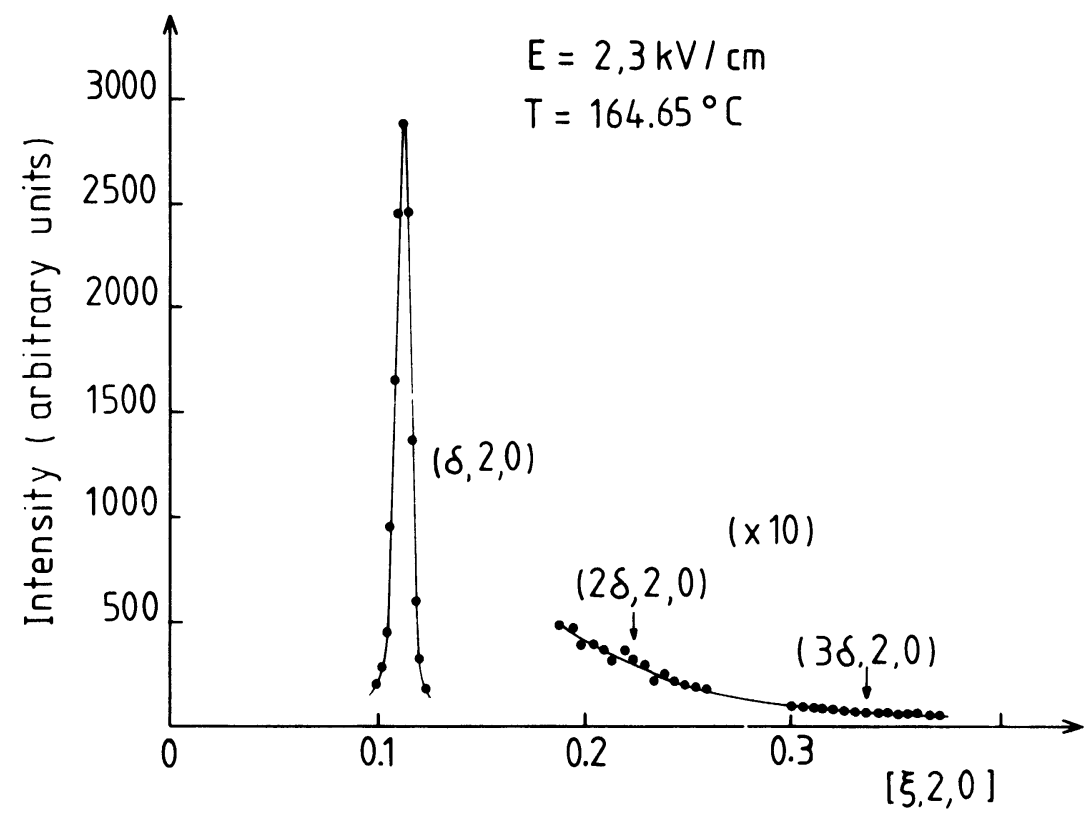

Fig. 3. - Attempt to observe higher-order satellites under field $\left(E=2.3 \mathrm{kV} \mathrm{cm}{ }^{-1}, T=164.65^{\circ} \mathrm{C}\right)$. The background intensity corresponds to diffuse quasielastic scattering which persists throughout the incommensurate region.

4. Model. - 4.1 Form OF THE FREe-ENERgy. - The phase diagram in figure 2 is best discussed in terms of a free-energy functional involving the component of the polarization along $\mathbf{b}$, $P_{y}$, and its spatial derivatives along a :

$G=\int\left\{\frac{1}{2} A_{0} P_{y}^{2}+\frac{1}{4} B P_{y}^{4}+\frac{1}{6} C P_{y}^{6}+\frac{1}{2} \alpha\left(\frac{\mathrm{d} P_{y}}{\mathrm{~d} x}\right)^{2}+\frac{1}{4} \beta\left(\frac{\mathrm{d}^{2} P_{y}}{\mathrm{~d} x^{2}}\right)^{2}+\gamma\left(P_{y} \frac{\mathrm{d} P_{y}}{\mathrm{~d} x}\right)^{2}-E P_{y}\right\} \mathrm{d} x$.

General free-energy functionals of the above type have been considered by Hornreich et al. [20], Michelson [21], and Coutinho-Filho et al. [22] and, in the specific context of uniaxial ferroelectrics, by Ishibashi et al. [8] and Lederer et al. [23].

Following the authors of reference [8] we have included a term proportional to $\left(P_{y} \frac{\mathrm{d} P_{y}}{\mathrm{~d} x}\right)^{2}$. This term is essential for the overall consistency of the model, as discussed below. On the other hand our model differs from that of reference [8] in two important respects :

i) We assume $B<0$. The opposite assumption $(B>0)$ is not consistent with our observation 
of a direct para-ferro transition above $E_{\mathrm{T}}$. Furthermore, it is not consistent with the dielectric behaviour of $\mathrm{NaNO}_{2}$ in zero-field. For example, two different Curie laws are observed [1] in the paraelectric and ferroelectric phases :

$$
\chi_{22}^{\mathrm{p}, \mathrm{f}}=\frac{\mathcal{C}^{\mathrm{p}, \mathrm{f}}}{T-T_{\mathrm{c}}^{\mathrm{p}, \mathrm{f}}} .
$$

If the direct (virtual) para-ferro transition were continuous $(B>0)$, one would expect to find

$$
T_{\mathrm{c}}^{\mathrm{p}} \simeq T_{\mathrm{c}}^{\mathrm{f}} \simeq T_{\mathrm{f}}^{\prime}
$$

where $T_{\mathrm{f}}^{\prime}$ is the temperature of the virtual direct transition

$$
T_{\mathrm{f}}<T_{\mathrm{f}}^{\prime}<T_{\mathrm{i}}
$$

Instead Nomura [1] finds :

$$
T_{\mathrm{c}}^{\mathrm{f}}-T_{\mathrm{c}}^{\mathrm{p}} \simeq 3.5 \mathrm{~K}
$$

Similarly, the spontaneous polarization measured below $T_{\mathrm{f}}$, should extrapolate to zero at $T_{\mathrm{f}}^{\prime}$ (i.e. below $T_{\mathrm{i}}$ ). The data of Hamano [5] show that this is not possible, except through the use of an unrealistic critical exponent $(\beta=0.20)$.

ii) Following the discussion in $\S 3.3$, we assume a purely sinusoidal polarization wave, i.e. we write :

$$
P_{y}(x)=P_{0}+2 P_{\delta} \cos (2 \pi \delta x) .
$$

Inserting (5) into (4) and neglecting some unessential sixth-order terms, we get :

$$
G=\frac{1}{2} A_{0} P_{0}^{2}+\frac{1}{4} B P_{0}^{4}+\frac{1}{6} C P_{0}^{6}+A_{\delta} P_{\delta}^{2}+\frac{3}{2} B_{\delta} P_{\delta}^{4}+\frac{3}{2}\left(B+B_{\delta}\right) P_{0}^{2} P_{\delta}^{2}-P_{0} E
$$
with :

$$
A_{\delta}=A_{0}+\alpha \delta^{2}+\frac{1}{2} \beta \delta^{4}
$$

and :

$$
B_{\delta}=B+\frac{4}{3} \gamma \delta^{2}
$$

In addition, we assume :

$$
A_{0}=a\left(T-T_{0}\right) ; \quad B<0 ; \quad C>0 ; \quad \alpha<0 ; \quad \beta>0 ; \quad \gamma>0 .
$$

4.2 SinUSOIDAL PHASE IN ZERO-FIELD. - Setting $P_{0}=0$ in (6), the free-energy reduces to :

$$
G_{\mathrm{s}}=A_{\delta} P_{\delta}^{2}+\frac{3}{2} B_{\delta} P_{\delta}^{4} .
$$

The para-sinusoidal transition temperature $T_{\mathrm{i}}$ and the modulation wavevector at $T_{\mathrm{i}}, \delta_{\mathrm{i}}$, are obtained from :

$$
A_{\delta}=\frac{\partial A_{\delta}}{\partial \delta}=0
$$

which yields :

$$
\left.\begin{array}{ll}
\delta_{\mathrm{i}}^{2}=-\frac{\alpha}{\beta}, & (a) \\
a\left(T_{\mathrm{i}}-T_{0}\right)=\frac{\alpha^{2}}{2 \beta} & (b)
\end{array}\right\}
$$


In order to have a continuous transition at $T_{\mathrm{i}}$ we have implicitly assumed $B_{\delta_{\mathrm{i}}}\left(\equiv B_{\mathrm{i}}\right)>0$, which in turn implies :

$$
\frac{4}{3} \gamma \delta_{i}^{2}>|B|
$$

Below $T_{\mathrm{i}}$, (9) may be minimized firstly with respect to the amplitude $P_{\delta}$ of the modulation, yielding :

$$
G_{\mathrm{s}}=-\frac{A_{\delta}^{2}}{6 B_{\delta}}
$$

and secondly with respect to the value of the modulation wavevector $\delta$ :

$$
\frac{\partial G_{\mathrm{s}}}{\partial \delta}=0
$$

With the help of (7), (8) and (10), equation (13) yields :

$$
\delta^{2}-\delta_{\mathrm{i}}^{2}=\frac{1}{2}\left\{\left[\frac{B_{\mathrm{i}}^{2}}{\gamma^{2}}+\frac{8 a}{3 \beta}\left(T-T_{\mathrm{i}}\right)\right]^{1 / 2}-\frac{B_{\mathrm{i}}}{\gamma}\right\} .
$$

Close to $T_{\mathrm{i}}$ we have :

$$
\delta^{2}-\delta_{\mathrm{i}}^{2} \simeq \mu\left(T-T_{\mathrm{i}}\right)
$$

With :

$$
\mu=\frac{2 \gamma a}{3 \beta B_{\mathrm{i}}} .
$$

Finally the zero-field free-energy of the sinusoidal phase is obtained as

$$
G_{\mathrm{s}}(T)=-\frac{a^{2}\left(T-T_{\mathrm{i}}\right)^{2}}{6 B_{\mathrm{i}}}+\mathcal{O}\left(T-T_{\mathrm{i}}\right)^{3} .
$$

4.3 Para-SinUsoidal transition Line $\left(T_{\mathrm{i}}(E)\right)$. - Assuming $B_{\delta_{\mathrm{i}}}(E)>0$, the equation of the transition line is obtained by setting to zero the coefficient of $P_{\delta}^{2}$ in (6) :

$$
A_{\delta}+\frac{3}{2}\left(B+B_{\delta}\right) P_{0}^{2}=0 .
$$

The value of $\delta_{\mathrm{i}}(E)$ is obtained by taking the derivative of (17) with respect to $\delta$. Whence :

$$
\delta_{\mathrm{i}}^{2}(E)-\delta_{\mathrm{i}}^{2}=-\frac{2 \gamma}{\beta} P_{0}^{2}
$$

In the non-ferroelectric state we have :

$$
P_{0}=\frac{E}{A_{0}}\left(1-\frac{B E^{2}}{A_{0}^{3}}\right)+\mathcal{O}\left(E^{5}\right)
$$

For $E \ll\left(\frac{A_{0}^{3}}{|B|}\right)^{1 / 2}$ we may take

$$
P_{0} \simeq \frac{E}{A_{0}},
$$


whence :

with :

$$
\delta_{\mathrm{i}}^{2}(E)-\delta_{\mathrm{i}}^{2}=\lambda E^{2}+\mathcal{O}\left(E^{4}\right)
$$

$$
\lambda=-\frac{2 \gamma}{\beta a^{2}\left(T_{\mathrm{i}}-T_{0}\right)^{2}} .
$$

Finally, the equation of the transition line is obtained as :

$$
a\left(T_{\mathrm{i}}(E)-T_{\mathrm{i}}\right)=-\frac{3}{2}\left(B+B_{\mathrm{i}}\right) \frac{E^{2}}{a^{2}\left(T_{\mathrm{i}}-T_{0}\right)^{2}}+\mathcal{O}\left(E^{4}\right) .
$$

Combining (15) and (19) the quantity $B_{\mathrm{i}}$ appearing in (20), is expressed in terms of observable quantities as :

$$
B_{\mathrm{i}}=-\frac{a^{3}}{3}\left(T_{\mathrm{i}}-T_{0}\right)^{2} \frac{\lambda}{\mu} \text {. }
$$

4.4 Direct PaRA-FerRo TRANSITION LINE $\left(T_{\mathrm{f}}^{\prime}(E)\right)$. - The stability of the ferroelectric phase with respect to the paraelectric phase is discussed using equation (6) with $P_{\delta}=0$. The coexistence line originates at the point $\left(E=0, T=T_{\mathrm{f}}^{\prime}\right)$ and ends at the critical point $\left(E_{\mathrm{c}}, T_{\mathrm{c}}\right)$, with

$$
\begin{aligned}
& a\left(T_{\mathrm{f}}^{\prime}-T_{0}\right)=\frac{3 B^{2}}{16 C} \\
& a\left(T_{\mathrm{c}}-T_{0}\right)=\frac{9 B^{2}}{20 C} ; \quad E_{\mathrm{c}}=\frac{8 C}{3}\left(\frac{-3 B}{10 C}\right)^{5 / 2} .
\end{aligned}
$$

The slope of the line at $E=0$ and $E=E_{\mathrm{c}}$ is given by

and

$$
\left(\frac{\mathrm{d} E}{\mathrm{~d} T}\right)_{E=0}=\frac{1}{2} a\left(\frac{-3 B}{4 C}\right)^{1 / 2},
$$

$$
\left(\frac{\mathrm{d} E}{\mathrm{~d} T}\right)_{E_{\mathrm{c}}}=a\left(\frac{-3 B}{10 C}\right) .
$$

The transition at $T_{\mathrm{f}}^{\prime}(E)$ is virtual below the triple point and real above.

4.5 Ferro-SinUsoidal TRANSition Line $\left(T_{\mathrm{f}}(E)\right)$. - The difference $T_{\mathrm{f}}^{\prime}(E)-T_{\mathrm{f}}(E)$ is largest at $E=0$ and vanishes at $E_{\mathrm{T}}$. Furthermore, a simple thermodynamic argument shows that if, as is the case here, the transition at $T_{\mathrm{i}}(E)$ remains continuous all the way up to $E_{\mathrm{T}}$, the two curves $T_{\mathrm{f}}(E)$ and $T_{\mathrm{f}}^{\prime}(E)$ should have a common tangent at $E_{\mathrm{T}}$.

The zero field transition temperature $T_{\mathrm{f}}$ is obtained by equating the free energies of the ferro and sinusoidal phases :

$$
G_{\mathrm{s}}\left(T_{\mathrm{f}}\right)=G_{\mathrm{f}}\left(T_{\mathrm{f}}\right)
$$

where $G_{\mathrm{s}}$ is given in (12), while $G_{\mathrm{f}}$ is obtained from (2) :

$$
G_{\mathrm{f}}(T)=\frac{1}{2} a\left(T-T_{0}\right) P_{0}^{2}+\frac{1}{4} B P_{0}^{4}+\frac{1}{6} C P_{0}^{6} .
$$

Minimizing (23) with respect to the spontaneous polarization $P_{0}$ we get :

$$
P_{0}^{2}=\frac{|B|+\sqrt{B^{2}-4 a C\left(T-T_{0}\right)}}{2 C} .
$$


For realistic values of the coefficients $a, T_{0}, B, B_{\mathrm{i}}$ and $C$ (cf. $\S 5$ ), the numerical solution of (22) yields :

$$
T_{\mathrm{f}}^{\prime}-T_{\mathrm{f}} \simeq 0.1 \mathrm{~K} \text {. }
$$

This small temperature difference reflects the large entropy difference between the ferroelectric state on one hand and the paraelectric and sinusoidal states on the other hand.

5. Discussion. - The phenomenological model developed in the previous section is readily applied to the case of $\mathrm{NaNO}_{2}$. Starting from the known values [4] of the paraelectric Curie constant and Curie temperature $\left(\mathrm{C}=5130 \mathrm{~K} ; T_{\mathrm{i}}-T_{0}=2.45 \mathrm{~K}\right)$ and from the observed value of $\delta_{\mathrm{i}}(=0.122$ red. units), we obtain, with the help of $(10)$ :

$$
\begin{aligned}
& a=2.2 \times 10^{7} \mathrm{MKS}, \\
& \left.\alpha=-7.3 \times 10^{9} \mathrm{MKS} \text { (r.u.) }\right)^{-2}, \\
& \left.\beta=4.9 \times 10^{11} \mathrm{MKS} \text { (r.u. }\right)^{-4} .
\end{aligned}
$$

From the observed values of $\lambda$ and $\mu\left(\lambda=1.6 \times 10^{-4} \text { (r.u. }\right)^{2} \mathrm{kV}^{-2} \mathrm{~cm}^{2} ; \mu=0.22 \times 10^{-2}$ (r.u. $\left.)^{2} \mathrm{~K}^{-1}\right)$ and using (19) and (21) we get :

$$
\begin{aligned}
\gamma & =1.15 \times 10^{13} \mathrm{MKS}(\text { r.u. })^{-2}, \\
B_{\mathrm{i}} & =1.55 \times 10^{11} \mathrm{MKS},
\end{aligned}
$$

whence :

$$
B=B_{\mathrm{i}}-\frac{4}{3} \gamma \delta_{\mathrm{i}}^{2}=-0.70 \times 10^{11} \mathrm{MKS} .
$$

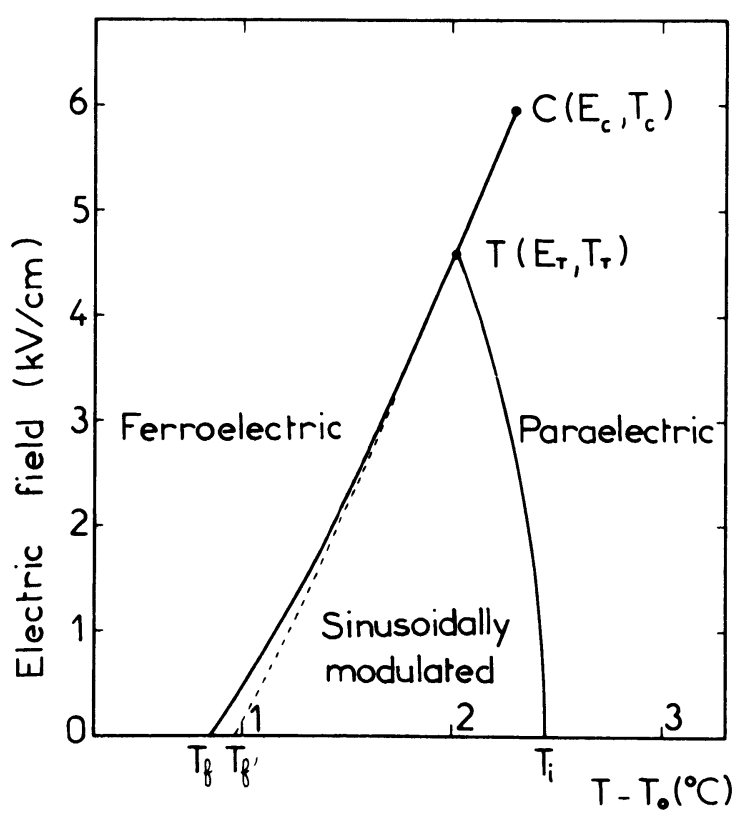

Fig. 4. - Calculated $(E, T)$ phase diagram. The model is constrained to yield the correct value of $T_{\mathrm{i}}-T_{\mathrm{f}}$. The virtual transition line $T_{\mathrm{f}}^{\prime}(E)$ is shown as a dotted line. 
Finally, fixing the value of $T_{\mathrm{f}}\left(T_{\mathrm{i}}-T_{\mathrm{f}}=1.6 \mathrm{~K}\right)$ we determine the value of $C$ :

$$
C=4.3 \times 10^{13} \mathrm{MKS} .
$$

Figure 4 shows the phase diagram calculated using the above numerical values. The agreement with the observed phase diagram is only semi-quantitative. In particular the model underestimates the downward bending of the $T_{\mathrm{i}}(E)$ line. This discrepancy is only partly due to our neglect of terms of order $E^{4}$ in deriving(20).

Finally, since none of the observables used in the calculation depend upon the value of $k$ in equation (1), we can use the calculated phase diagram to estimate the uncertainty on the temperature scale arising from our identification of the $T_{\mathrm{f}}(E)$ line to a straight line : we find an uncertainty of about $0.1 \mathrm{~K}$ at $E=E_{\mathrm{T}}$.

In conclusion we find that the simple phenomenological model presented here gives a good semi-quantitative account of the observed phase diagram. In this model the term $\propto\left(P_{y} \frac{\partial P_{y}}{\partial x}\right)^{2}$ plays a central role in so far as it accounts for the temperature and field dependence of the modulation wavevector. At the same time it explains why the transition at $T_{\mathrm{i}}$ is found to be continuous, while the direct para-ferro transition would be discontinuous. Obviously, more elaborate treatments are possible and are probably warranted on the basis of the present data. Further experimental work is underway in order to determine the position of the critical point.

Acknowledgments. - We wish to acknowledge the technical assistance of L. Deschamps, R. Bertrand and A. Vaures for sample preparation and of D. Brochier for his contribution to the design of the furnace. We are grateful to J. P. Chapelle for the loan of his excellent single-crystal and to M. Lambert, G. André, J. Lajzerowicz, M. Vallade, G. Dolino and D. Mukamel for stimulating discussions.

\section{References}

[1] Nomura, S., J. Phys. Soc. Japan 16 (1961) 2440.

[2] Yamada, Y., ShibuYa, I. and Hoshino, S., J. Phys. Soc. Japan 18 (1963) 1594.

[3] Sakurai, J., Cowley, R. A. and Dolling, G., J. Phys. Soc. Japan 28 (1970) 1426.

[4] Hatta, I., J. Phys. Soc. Japan 28 (1970) 1266.

[5] Hamano, K., J. Phys. Soc. Japan 35 (1973) 157.

[6] Yamada, Y. and Yamada, T., J. Phys. Soc. Japan 21 (1966) 2167.

[7] Levanyuk, A. P. and Sannikov, D. G., Sov. Phys. Solid State 18 (1976) 1122.

[8] Ishibashi, Y. and Shiba, H., J. Phys. Soc. Japan 45 (1978) 409.

[9] Michel, K. H., Phys. Rev. B 24 (1981) 3998.

[10] Heine, V. and McConnell, J. D. C., Phys. Rev. Lett. 46 (1980) 1092.

[11] Klein, M. L., McDonald, I. R. and OzaKi, Y., Phys. Rev. Lett. 48 (1982) 1197.

[12] Aizu, J. Phys. Soc. Japan 51 (1982) 515 ; 51 (1982) 2885.

[13] Durand, D., Denoyer, F., Lambert, M., Bernard, L. and Currat, R., J. Physique 43 (1982) 149.

[14] Moudden, A. H., Denoyer, F. and Lambert, M., J. Physique 39 (1978) 1323.

[15] Denoyer, F., Moudden, A. H., Currat, R., Vettier, C., Bellamy, A. and Lambert, M., Phys. Rev. B 25 (1982) 1697.

[16] JAMET, J. P., J. Physique-Lett. 42 (1981) L-123.

[17] Jamet, J. P., Lederer, P. and Moudden, A. H., Phys. Rev. Lett. 48 (1982) 442.

[18] Moudden, A. H., Svensson, E. C. and Shirane, G., Phys. Rev. Lett. 49 (1982) 557.

[19] Gesi, K., Japan J. Applied Phys. 4 (1965) 818.

[20] Hornreich, R. M., Luban, M. and Shtrickman, S., Phys. Rev. Lett. 35 (1975) 1678.

[21] Michelson, A., Phys. Rev. B 16 (1977) 577.

[22] Coutinho-Filho, M. E. and de Moura, M. A., J. Magn. Magn. Mat. 15-18 (1980) 433.

[23] Lederer, P. and Chaves, C. M., J. Physique-Lett. 42 (1981) L-127. 